\title{
Multimodal imaging findings in a patient with type I sialidosis with a compound heterozygous mutation in the NEU1 gene
}

\author{
Chao Wan ${ }^{1 \#}$, Meina Lin ${ }^{2 \#}$, Miao Jiang ${ }^{2}$, Rui Hua ${ }^{1}$ \\ ${ }^{1}$ Department of Ophthalmology, First Hospital of China Medical University, Shenyang, China; ${ }^{2}$ Key Laboratory of Reproductive Health, Liaoning \\ Province Research Institute of Family Planning, China Medical University, Shenyang, China \\ \#These authors contributed equally to this work. \\ Correspondence to: Miao Jiang, MD. Key Laboratory of Reproductive Health, Liaoning Province Research Institute of Family Planning, China Medical \\ University, Huanggu District, Shenyang 110000, China. Email: jiangmiaocpt@aliyun.com; Rui Hua, MD. Department of Ophthalmology, The First \\ Affiliated Hospital of China Medical University, No. 155 Nanjingbei Street, Heping District, Shenyang 110001, China. Email: woodshua@126.com.
}

Submitted Jul 21, 2020. Accepted for publication Jan 18, 2021.

doi: 10.21037/qims-20-891

View this article at: http://dx.doi.org/10.21037/qims-20-891

\section{Introduction}

Mutations in the neuraminidase 1 (NEU1) gene, which encodes the enzyme neuraminidase, lead to an autosomal recessive lysosomal storage disease, known as sialidosis (1). Patients with type I sialidosis usually have mild symptoms, such as the typical cherry-red spot myoclonus syndrome with visual impairment, myoclonic epilepsy, and ataxia in the second or third decade of life (2). Sialidosis is a very rare disease, with an incidence of approximately 1/4,000,000 live births in the Caucasian population (3). Only five cases of type I sialidosis with macular cherry-red spots have been reported in the Chinese population (4-6). Moreover, secondary optic disc abnormalities have not been observed in Chinese patients.

Herein, we present the macula and optic disc's multimodal imaging findings in a Chinese patient with type I sialidosis and a compound heterozygous NEU1 mutation during a 1-year follow-up.

\section{Case report}

An 11-year-old girl with normal intelligence complained of blurred binocular vision for 3 years. She was born by normal delivery from non-consanguineous parents. The best-corrected visual acuity was $48 / 60$ on the right and $36 / 60$ on the left. Transparent corneas and lenticular opacities were found in the bilateral anterior segments.
Fundus examination of the bilateral eyes revealed macular cherry-red spots with surrounding opacities, and the cupto-disc ratio was approximately 0.4 at the optic nerve head, with a normal appearance.

Multicolor imaging (MCI) highlighted the bilateral macular cherry-red spots with a distinct boundary. The optical coherence tomography (OCT) posterior pole asymmetry analysis (PPAA) showed increased parafoveal ganglion cell layers bilaterally. B-scan OCT revealed increased reflectivity in the macular ganglion cell layer region without a clear boundary. There was apparent hyperreflectivity of the foveal region photoreceptor layer (Figure 1). OCT with Bruch's membrane opening minimum rim width (BMO-MRW) parameter showed circumpapillary retinal nerve fiber layer (cpRNFL) atrophy (Figure 2). The mean BMO-MRW values in the reference database (ageand BMO area-adjusted), according to the manufacturer's information, were as follows: $336.1 \pm 51.63 \mu \mathrm{m}$ (BMO-MRW Global), 238.7 $\pm 42.83 \mu \mathrm{m}$ (BMO-MRW T); $321.1 \pm 59.87 \mu \mathrm{m}$ (BMO-MRW TS); 352.4 $\pm 60.25 \mu \mathrm{m}$ (BMO-MRW TI); 374.2 $\pm 63.67 \mu \mathrm{m}$ (BMO-MRW N); $374.2 \pm 71.09 \mu \mathrm{m}$ (BMOMRW NS); and 411.6 $\pm 71.87 \mu \mathrm{m}$ (BMO-MRW NI). Irregular visual field defects were identified in the $30^{\circ}$ visual field bilaterally. For the right eye, the mean deviation (MD) was -13.39 decibels $(\mathrm{dB})$, and for the left eye, the MD was $-16.72 \mathrm{~dB}$ (normal MD value: $0 \mathrm{~dB}$ ). Pattern visual evoked potentials were delayed, as P100 was 113 ms in both eyes 


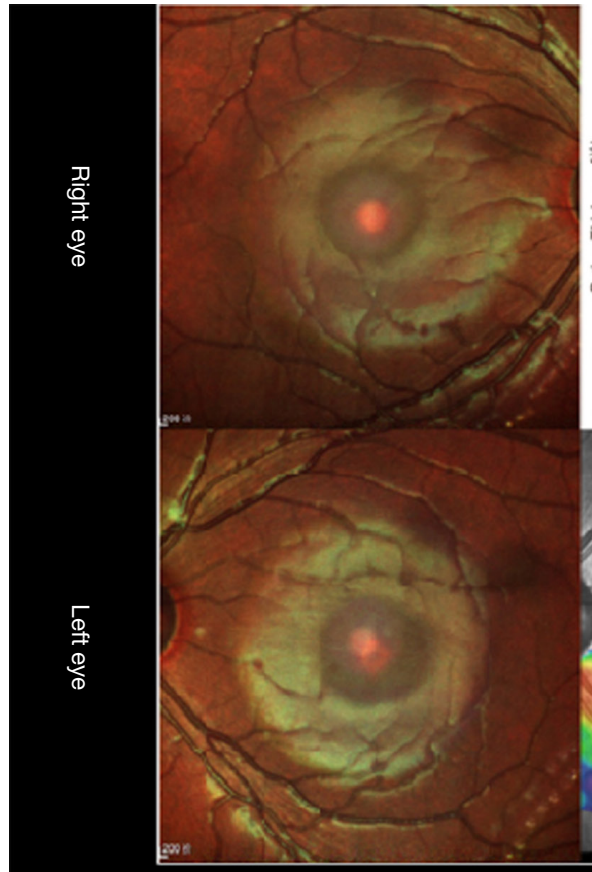

$\mathrm{MCl}$

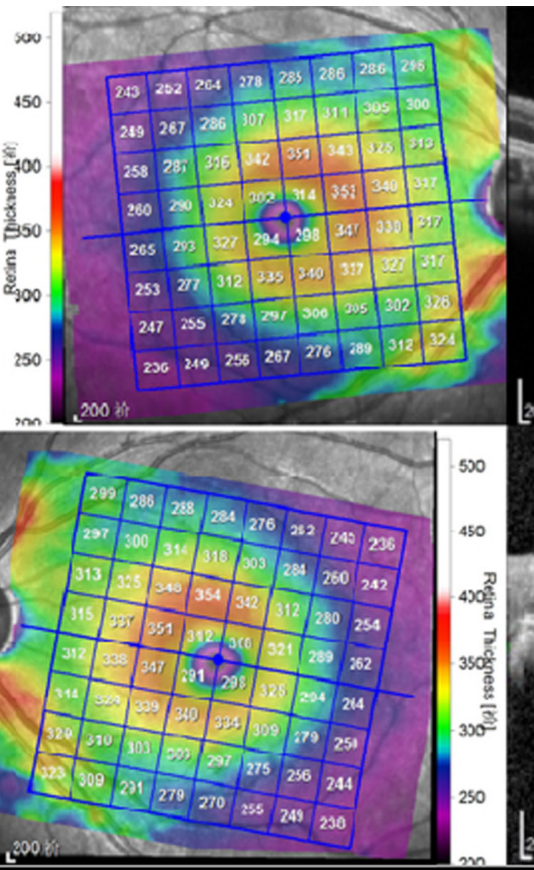

PPAA

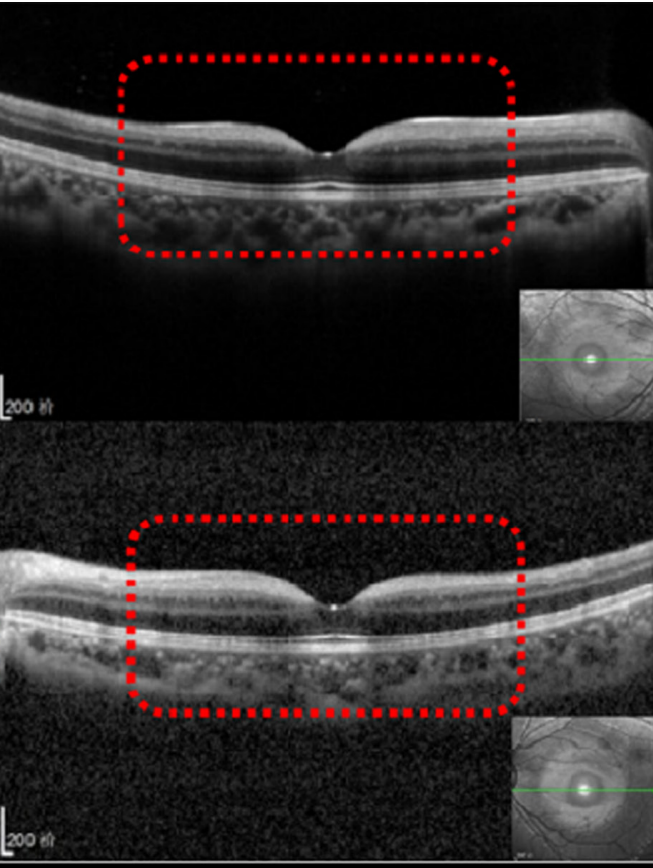

OCT

Figure 1 Multimodal imaging results (MCI, PPAA, OCT). MCI showed obvious central macular cherry-red spots with a distinct boundary bilaterally. PPAA indicated increased parafoveal ganglion cell layers in both eyes. OCT demonstrated both increased reflectivity in the macular ganglion cell layer and hyperreflectivity of the photoreceptor layer in the foveal region (red dotted box; the illustration shows the OCT profile orientation). MCI, multicolor imaging; PPAA, posterior pole asymmetry analysis; OCT, optical coherence tomography.

(normal range: $81-105 \mathrm{~ms}$ ), indicating bilateral visual pathway dysfunction.

Moreover, the patient developed ataxia at 9 years of age. Her 28-year-old sister, who had similar symptoms since 18 , was now bedridden and had epilepsy.

Sanger sequencing analysis of the NEU1 gene (NM_000434) revealed a c.239C >T (p.P80L) and c.544A>G (p.S182G) compound heterozygous mutation in the patient. Accordingly, we tested other family members for these two mutations. The patient's sister had the same compound heterozygous mutation, c.239C > T (p.P80L) and c.544A $>\mathrm{G}$ (p.S182G), her mother had a c.239C >T heterozygous mutation, and her father had a c.544A>G heterozygous mutation. The parents had no clinical symptoms of type I sialidosis.

Based on all results, we diagnosed the patient with type I sialidosis. Because there is currently no effective treatment, the patient was only followed-up by ophthalmologists and neurologists. After 1 year, OCT with BMO-MRW showed persistent cpRNFL atrophy in both eyes, compared with the initial diagnosis findings. En face OCT showed diffuse enhanced reflection from the ganglion cell layer bilaterally. However, OCT angiography (OCTA) revealed normal retinal vasculature bilaterally (Figure 1 ).

All procedures performed were following the ethical standards of the institutional and/or national research committee(s) and with the Declaration of Helsinki (as revised in 2013). Written informed consent was obtained from the patient and her parents to publish this case report and any accompanying images.

\section{Discussion}

In this study, we confirmed a compound heterozygous mutation, c.239C >T (p.P80L) and c.544A >G (p.S182G), in two siblings with type I sialidosis and described the related multimodal imaging findings.

According to the Human Gene Mutation Database Professional, release 2019.3 (http://www.hgmd.cf.ac.uk/ ac/all.php), over 45 mutations in the NEU1 gene have 


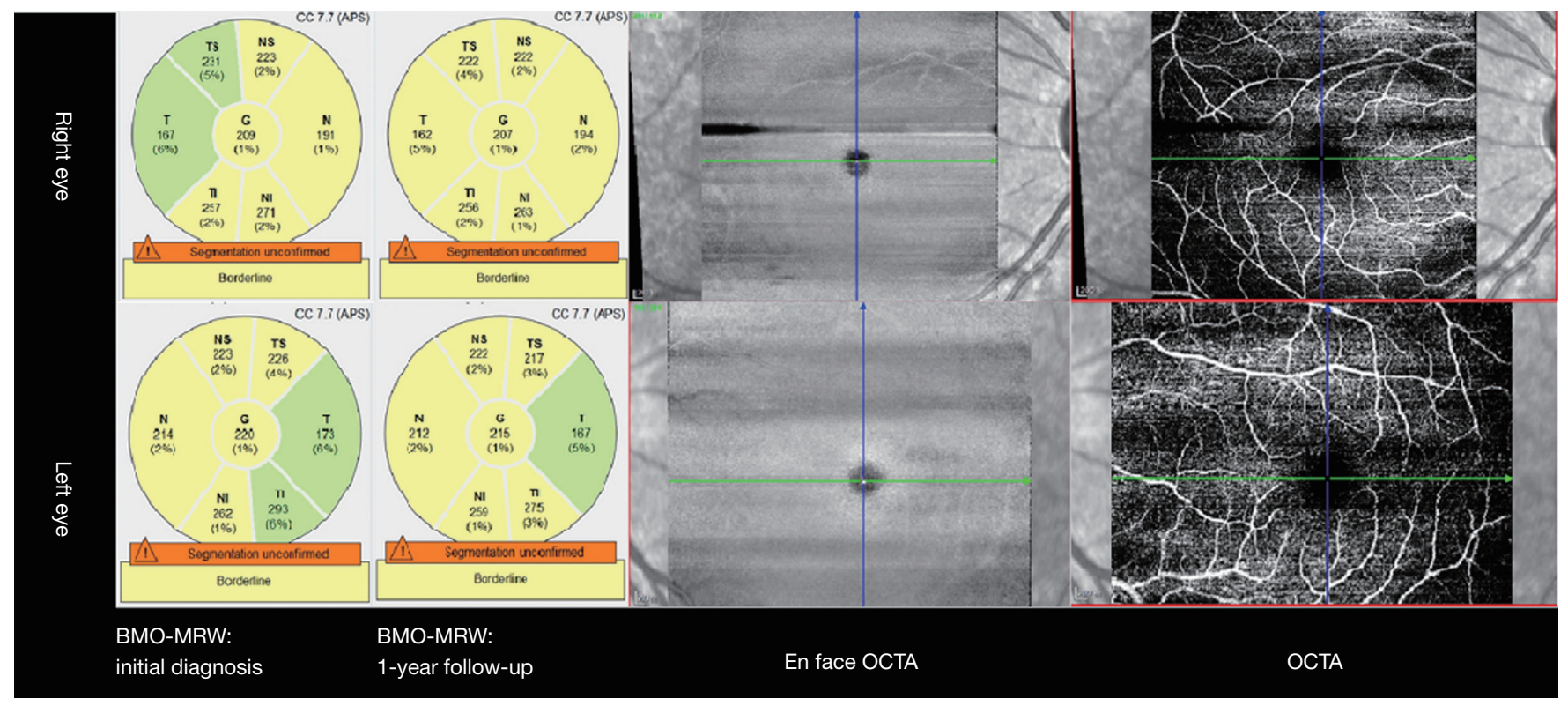

Figure 2 Multimodal imaging results (BMO-MRW, en face OCT, OCTA). OCT with BMO-MRW parameter showed persistent cpRNFL sectional atrophy in both eyes. The normal BMO-MRW values are as follows: $336.1 \pm 51.63 \mu \mathrm{m}(\mathrm{BMO}-\mathrm{MRW} \mathrm{Global}), 238.7 \pm 42.83 \mu \mathrm{m}$ (BMO-MRW T); 321.1 $\pm 59.87 \mu \mathrm{m}$ (BMO-MRW TS); 352.4 $460.25 \mu \mathrm{m}$ (BMO-MRW TI); 374.2 $\pm 63.67 \mu \mathrm{m}$ (BMO-MRW N); $374.2 \pm 71.09 \mu \mathrm{m}$ (BMO-MRW NS); and 411.6 $\pm 71.87 \mu \mathrm{m}$ (BMO-MRW NI). En face OCT revealed diffuse enhanced reflection from the ganglion cell layer in both eyes. OCTA demonstrated normal retinal vasculature in the ganglion cell layer bilaterally. BMO-MRW, Bruch's membrane opening minimum rim width; OCT, optical coherence tomography; OCTA, OCT angiography; cpRNFL, circumpapillary retinal nerve fiber layer.

been identified to date. The NEU1 mutation c.239C>T (p.P80L) was previously identified in a patient with type II sialidosis with extremely severe symptoms, who died after birth (7). This patient had a compound heterozygous mutation of p.P80L and p.W240R. The P80 residue is situated in a conserved RIP motif (Arg-Ile/Leu-Pro) adjacent to the active center, and the $\mathrm{P} 240$ residue is situated in a conserved ASP box (SerX-Asp-X-Gly-X-ThrTrp) of the NEU1 protein. Therefore, these mutations' hypothetical consequences (p.P80L and p.W240R) would be conformational changes of the protein, leading to loss of enzymatic activity. Accordingly, the extremely severe symptoms in this patient were presumably due to absent enzymatic activity.

The homozygous NEU1 mutation c.544A>G (p.S182G) was first reported in a Chinese patient with type I sialidosis with relatively mild symptoms, and in vitro experiments revealed that the p.S182G mutant had $20-40 \%$ of normal sialidase activity, which may be because the mutation is located at the base of the long flexible surface loop (8). Therefore, this patient had relatively mild symptoms due to the residual sialidase activity. The present case is the first report of the c.239C>T (p.P80L) and c.544A>G (p.S182G) compound heterozygous mutation in the NEU1 gene in type I sialidosis. The patient's relatively mild symptoms were most likely due to the p.S182G mutant's residual enzymatic activity.

The typical macular cherry-red spots are seen in type I sialidosis always lead to a misdiagnosis of central retinal artery occlusion. The lipofuscin granule deposition in the perifoveal ganglion cell layer results in a white patch around the fovea. In contrast, because it lacks ganglion cells, the foveal pit maintains its original reddish appearance (4). MCI with both short- and long-wavelength scanning lasers enhanced the distinct contrast appearance of the macular cherry-red spots in our patient. Compared with the macular cherry-red spots in central retinal artery occlusion (9), OCTA showed normal retinal vasculature, which was helpful for differential diagnosis.

Kersten et al. (3) reported that OCT scans showed cpRNFL thickening due to abnormal metabolic product accumulation. However, in our study, OCT with BMOMRW showed sectional atrophy of the cpRNFL, corresponding with the visual field abnormalities. This is 
the first report of optic nerve atrophy in type I sialidosis. Moreover, we observed persistent aggravation of the optic disc atrophy during the 1-year follow-up.

In conclusion, this is the first report to describe the OCTA, MCI, and BMO-MRW findings in a patient with type I sialidosis with a compound heterozygous mutation in the NEU1 gene, contributing to the comprehensive understanding of this rare disease.

\section{Acknowledgments}

Funding: This work was supported by grants from the Fund for Clinical Genetics (Ophthalmology) of the Subject Construction Project of China Medical University. The funders had no role in the study design, data collection, analysis, decision to publish, or preparation of the manuscript.

\section{Footnote}

Conflicts of Interest: All authors have completed the ICMJE uniform disclosure form (available at http://dx.doi. org/10.21037/qims-20-891). The authors have no conflicts of interest to declare.

Ethical Statement: The authors are accountable for all aspects of the work in ensuring that questions related to the accuracy or integrity of any part of the work are appropriately investigated and resolved. All procedures performed were following the ethical standards of the institutional and/or national research committee(s) and with the Declaration of Helsinki (as revised in 2013). Written informed consent was obtained from the patient and her parents to publish this case report and any accompanying images.

Open Access Statement: This is an Open Access article distributed in accordance with the Creative Commons Attribution-NonCommercial-NoDerivs 4.0 International License (CC BY-NC-ND 4.0), which permits the noncommercial replication and distribution of the article with the strict proviso that no changes or edits are made and the original work is properly cited (including links to both the formal publication through the relevant DOI and the license). See: https://creativecommons.org/licenses/by-nc-nd/4.0/.

\section{References}

1. Bonten E, van der Spoel A, Fornerod M, Grosveld
G, d'Azzo A. Characterization of human lysosomal neuraminidase defines the molecular basis of the metabolic storage disorder sialidosis. Genes Dev 1996;10:3156-69.

2. Canafoglia L, Robbiano A, Pareyson D, Panzica F, Nanetti L, Giovagnoli AR, Venerando A, Gellera C, Franceschetti S, Zara F. Expanding sialidosis spectrum by genome-wide screening: NEU1 mutations in adult-onset myoclonus. Neurology 2014;82:2003-6.

3. Kersten HM, Roxburgh RH, Danesh-Meyer HV, Hutchinson DO. Optical coherence tomography findings in a patient with type 1 sialidosis. J Clin Neurosci 2016;31:199-201.

4. Zou W, Wang X, Tian G. Fundus autofluorescence and optical coherence tomography of a macular cherryred spot in a case report of sialidosis. BMC Ophthalmol 2016;16:30.

5. Lai SC, Chen RS, Wu Chou YH, Chang HC, Kao LY, Huang YZ, Weng YH, Chen JK, Hwu WL, Lu CS. A longitudinal study of Taiwanese sialidosis type 1: an insight into the concept of cherry-red spot myoclonus syndrome. Eur J Neurol 2009;16:912-9.

6. Wang IH, Lin TY, Kao ST. Optical coherence tomography features in a case of Type I sialidosis. Taiwan J Ophthalmol 2017;7:108-11.

7. Itoh K, Naganawa Y, Matsuzawa F, Aikawa S, Doi H, Sasagasako N, Yamada T, Kira J, Kobayashi T, Pshezhetsky AV, Sakuraba H. Novel missense mutations in the human lysosomal sialidase gene in sialidosis patients and prediction of structural alterations of mutant enzymes. J Hum Genet 2002;47:29-37.

8. Lukong KE, Elsliger MA, Chang Y, Richard C, Thomas G, Carey W, Tylki-Szymanska A, Czartoryska B, Buchholz T, Criado GR, Palmeri S, Pshezhetsky AV. Characterization of the sialidase molecular defects in sialidosis patients suggests the structural organization of the lysosomal multienzyme complex. Hum Mol Genet 2000;9:1075-85.

9. Damento G, Chen MH, Leng T. Spectral-domain optical coherence tomography angiography of central retinal artery occlusion. Ophthalmic Surg Lasers Imaging Retina 2016;47:467-70.

Cite this article as: Wan C, Lin M, Jiang M, Hua R. Multimodal imaging findings in a patient with type I sialidosis with a compound heterozygous mutation in the NEU1 gene. Quant Imaging Med Surg 2021;11(9):4219-4222. doi 10.21037/qims-20-891 